\title{
Role of lipids and free radicals in diabetic with hypertension and smokers for causation of coronary heart disease
}

\author{
Suresh S. Ugle ${ }^{1}$, Mahendra D. Bikkad ${ }^{2}$ \\ ${ }^{1}$ Dr. Suresh S. Ugle, ${ }^{2}$ Dr. Mahendra D. Bikkad, Both authors are attached with Department of Biochemistry, \\ MIMSR Medical College, Latur, Maharashtra, India
}

Address for Correspondence: Suresh S. Ugle, Dept. of Biochemistry, MIMSR Medical College, Ambajogai Road, Latur, Maharashtra, India. E-mail: uglesuresh@rediffmail.com

\begin{abstract}
Introduction: The major independent risk factors for the development of atherosclerosis are the plasma cholesterol concentration, triglyceride, cigarette smoking, hypertension and diabetes, which are by them self's risk factor for coronary heart disease. Cardiovascular disease (CVD) threatens to cripple India's workforce and stunt India's growth if timely and appropriate public health measures are not instituted. Hyperglycemia, hypertension and cigarette smoking depletes natural antioxidants and facilitates the production of reactive oxygen species (ROS) which has the ability to react with all biological molecules and exert cytotoxic effects on cellular components that promotes and accelerate atherosclerosis. Hence the present study was aimed to find out collective effect of NIDDM, hypertension and cigarette smoking on lipid profile and oxidative stress in progression of CHD events. Materials and methods: We studied 50 healthy and 50 diabetes with hypertension and or smokers patients (i. e. had two or more CHD risk factors) patients with matched for age and body mass index. Blood samples were drawn after an overnight fast. We estimated serum triglycerides, total cholesterol, HDL-c and LDL-c and VLDL- c values were calculated by Friedwald's equation. We also estimated the total serum lipid peroxides by reaction with thiobarbituric acid. Results: In the control group I mean values of total cholesterol were $180.21 \pm 18.13 \mathrm{mg} \%$, LDL-c $106.60 \pm 18.2 \mathrm{mg} \%$, serum triglycerides were $99.90 \pm 16.14 \mathrm{mg}$ $\%$, HDL-c were $53.83 \pm 16.42 \mathrm{mg} \%$ and in the group II serum triglycerides $217.9 \pm 19.1 \mathrm{mg} \%$ and were significantly increased as compared to group I. The serum HDL-c group II $29.6 \pm 8.07 \mathrm{mg} \%$ were significantly decreased as compared to group I ( $\mathrm{P}$ is $<0.05)$. The serum lipid peroxides in the group 2nd $(296.9 . \pm 60.10)$ were significantly increased as compared to group 1st (180.96 \pm 35.16$)$. Conclusion: It can be concluded that patients of NIDDM with hypertension, smoking have higher total serum cholesterol, serum triglycerides, serum lipid peroxides (MDA), LDL- c and VLDL-c with lower HDL- c. These can be corrected, treated by dietary and life style modification before the development of end organ damage. For realization of exact role of lipid profile and oxidative stress further higher level studies should be done.
\end{abstract}

Keywords: Diabetes, Hypertension, Smoking, Lipids, Free Radicals and Coronary Heart Disease.

\section{Introduction}

The major independent risk factors for the development of atherosclerosis are the plasma cholesterol concentration, triglyceride, cigarette smoking, hypertension and diabetes, which are by them self's risk factor for coronary heart disease [1]. Despite recent decline in cardiovascular mortality, atherosclerotic disease is still major health problem facing Western society. Incidence of coronary heart disease has shown upward trends

Manuscript received: $06^{\text {th }}$ June 2017

Reviewed: $14^{\text {th }}$ June 2017

Author Corrected: $18^{\text {th }}$ June 2017

Accepted for Publication: 30 $0^{\text {th }}$ June 2017 in Indians in last decade [2]. The presence of any risk factor is associated with doubling the relative risk of developing atherosclerotic coronary artery disease. Cardiovascular disease (CVD) threatens to cripple India's workforce and stunt India's growth if timely and appropriate public health measures are not instituted.

A large amount of epidemiological evidences also supports the relationship between serum low density lipoprotein cholesterol (LDL-c) and 
coronary artery disease (CAD) in Indians [3]. Serum high density lipoprotein-cholesterol (HDLc) level has been found to have inverse relationship with the coronary artery disease (CAD) [4]. Diabetes mellitus is a common among Indians with coronary heart disease (CHD) both in their land of origin and abroad [5]. Individuals with non insulin dependent diabetes mellitus (NIDDM) are more likely to have multiple risk factors for CHD than age matched non diabetic subjects. Peoples with diabetes have a risk of CHD two to five times that of nondiabetic individuals [6]. Ttriglycerides levels, overweight and plasma triglyceride to HDLc ratio of three or greater are reliable indicator of insulin resistance and CHD [7]. NIDDM is associated with increase in plasma triglyceride and decrease in plasma HDL-c concentration i.e. dyslipidaemia changes that have been identified as the increasing risk of CHD.

Hypertension is a risk factor for the development of atherosclerosis. There is increasing evidence that atherosclerosis should be viewed fundamentally as an inflammatory disease. Atherogenic stimuli such as hyperlipidaemia appear to activate the inflammatory response by causing expression of mononuclear leukocyte recruiting mechanisms. There are several mechanisms which contribute to the development of vascular disease such as platelet disorders, abnormalities in lipoproteins and lipids i.e elevated total cholesterol, LDL-c, oxidative stress, oxidation of LDL, its deposition on endothelium and thus promotion of atherosclerosis. Both of hypertension and dyslipidaemia are independent risk factors for the development of atherosclerosis and known to have adverse effect on lipid profile and accelerate lipid peroxidation, there are limited studies to substantiate their cumulative effect on lipid profile and oxidative stress. It is estimated that the cigarette smoking habit directly contribute to above 3 million premature deaths each year. Although the relative risk of cigarrate smoking is great for lung cancer, the absolute risk is greatest for CHD and smoking related coronary deaths amounts to some 2.5 million yearly compared to 0.7 million for cancer [8]. Oxidative stress appears to be a probable clinically relevant factor in cigarette smoke related atherogensis and increased lipid peroxidation is also a risk factor for myocardial infarction [9]. Effect of smoking are dyslipidaemia, increased free radicals, oxidative stress, oxidation of LDL, damage to endothelium and thus promotion of atherosclerosis. Hyperglycemia, (NIDDM) hypertension and cigarette smoking depletes natural antioxidants and facilitates the production of reactive oxygen species (ROS) which has the ability to react with all biological molecules like lipids, proteins, carbohydrates, DNA etc and exert cytotoxic effects on cellular components [10].

Thus, increased ROS and impaired antioxidant defense contributes for initiation and progression of micro and macro vascular complications in diabetics hypertension and smoking [11]. Since the classic risk factors do not account for the excess risk of atherosclerosis in CHD risk factors, we need new approaches to explain the connection of this risk factors and accelerated vascular disease .Hence the present study was aimed to find out collective effect of NIDDM, hypertension and cigarette smoking on lipid profile and oxidative stress in progression of CHD events

\section{Materials and Methods}

Study design: This study was designed to investigate relationship between collective effect of NIDDM, hypertension and smoking on lipid profile and oxidative stress and to predicts its role in CHD events .We studied 50 healthy and 50 diabetes with hypertension and or smokers patients (i.e had two or more CHD risk factors) patients with matched for age and body mass index. Subjects were selected from medical, paramedical staff and general public who were around 40 to 60 year of age. All subjects were belonged to the Latur district of Marathwada region. Inclusion criteria for group 1 include healthy subject: The healthy subjects were nonsmokers, non obese, non alcoholic and free from any disease and not taking any drugs that alter lipid and carbohydrates metabolism

Patient belonging to group II were selected after attending medicine OPD of MIMSR Medical College, Latur and diagnosed as diabetic. All patients belonging to group II had NIDDM with hypertension and or smokers. Criteria of diagnosis of diabetes: The diagnosis of diabetes was based on American Diabetes association (ADA) [12] definition, i.e. fasting blood sugar levels $\geq 126$ $\mathrm{mg} \%$, Criteria for hypertension diagnosis: hypertension; systolic blood pressure greater than $140 \mathrm{~mm}$ of $\mathrm{Hg}$ and or diastolic blood pressure 90 $\mathrm{mm}$ of $\mathrm{Hg}$, and Smokers tobacco use; smoking 
more than ten cigarette or bidis per day for five or more years. All subjects after taking informed consent was interrogated and detailed examination was done. Blood samples drawn after an overnight fast. After serum separation the analysis was done on the same day. We estimated serum triglycerides by enzymatic method (Autopack Siemens kit) and total cholesterol by enzymatic methods (Autopack Siemens kit) HDL-c measured by phosphotung state method (Autopack Siemens kit). LDL-c and VLDL- c values were calculated by Friedwald's equation. We also estimated the total serum lipid peroxides by reaction with thiobarbituric acid using the method of Nadiger and Chandrakala [13]. Reaction involved in this method is malanodialdehyde combines with thiobarbituric acid to form malanodialdehydethiobarbituric acid complex which is measured colorimetrically at 530nm. The total serum lipid peroxides were calculated by using the coefficient of malanodialdehyde $1.5 \times 10^{5}$ and was expressed as malanodialdehyde /dl serum.

\section{Results}

Table-1: Serum Cholesterol and Other Biochemical Parameter in Normal Healthy Subject (Group I) and Diabetic with hypertension and or smokers patient (Group II)

\begin{tabular}{|l|l|l|l|l|l|l|l|}
\hline Group & $\begin{array}{l}\text { Total } \\
\text { cholesterol }\end{array}$ & $\begin{array}{l}\text { Triglyceri } \\
\text { des }\end{array}$ & HDL-C & LDL-C & VLDL-C & TC/HDL-C & $\begin{array}{l}\text { Serum Lipid } \\
\text { Peroxides } \\
\text { Mm/100ml }\end{array}$ \\
\hline 1st & $* 180.21$ & $* 99.90$ & $* 53.83$ & $* 106.60$ & $* 19.8$ & 3.6 & $* 180.96$ \\
N=50 & \pm 18.13 & \pm 16.14 & \pm 16.42 & \pm 18.2 & \pm 4.6 & \pm 1.01 & \pm 35.16 \\
\hline 2nd & $* 217.9$ & 208.14 & $\# 29.60$ & $* 147.21$ & $* 41.22$ & $* 7.75$ & $* 296.90$ \\
N=50 & \pm 19.1 & \pm 42.70 & \pm 8.07 & \pm 16.17 & \pm 8.30 & \pm 1.82 & \pm 60.10 \\
\hline
\end{tabular}

$*=\mathrm{P}<0.001, \#=\mathrm{P}<0.005$, The values are statistically significant.

In the control group I mean values of total cholesterol were $180.21 \pm 18.13 \mathrm{mg} \%$, LDL-c $106.60 \pm 18.2 \mathrm{mg} \%$, serum triglycerides were $99.90 \pm 16.14 \mathrm{mg} \%$, HDL-c were $53.83 \pm 16.42 \mathrm{mg} \%$ and in the group II serum triglycerides $217.9 \pm 19.1 \mathrm{mg} \%$ and were significantly increased as compared to group I. The serum HDL-c group II $29.6 \pm 8.07 \mathrm{mg} \%$ were significantly decreased as compared to group I ( $\mathrm{P}$ is $<0.05)$. The serum lipid peroxides in the group 2nd (296.9. \pm 60.10$)$ were significantly increased as compared to group $1^{\text {st }}(180 . .96 \pm 35.16)$.

\section{Discussion}

The catabolism of triglyceride-rich lipoproteins is initiated by lipoprotein lipase, an endothelial enzyme that hydrolyses the triglyceride moiety of chylomicrons and VLDL, and releases fatty acids for energy production in muscle and for storage in adipose tissue. Lipoprotein lipase activity is low in untreated or poorly controlled NIDDM and increase with improved glycaemic control [14]. In NIDDM passage of triglyceride- rich lipoproteins through the lipolytic cascade is delayed for two reasons: there is a shortage of catalytic sites on lipoprotein lipase, and over production of triglyceride saturates the sites that are available. Both mechanisms promote hypertriglyceridaemia. The two components of diabetic dyslipidaemia, high concentrations of triglyceride-rich lipoproteins and low concentrations of HDL, are closely interwoven. Hypertriglyceridaemia contributes to low HDL concentrations in one or combination of following reasons. 1) The first process involves the transfer of surface remnants'-redundant phospholipids and apolipoproteins from lipolysis of triglyceriderich lipoproteins - to HDL particles.Because lipoprotein lipase activity is decreased and lipolysis impaired in NIDDM, there are fewer surface remnants available to be incorporated into the HDL particle. 2)The large amount of triglyceride-rich lipoproteins and their prolonged residence time in the circulation increased the exchange of esterified cholesterol from HDL to triglyceride-rich lipoproteins and of triglyceride to HDL particles. The result is enrichment of the HDL particle core with triglyceride. Enriched HDL has a faster catabolic rate than normal HDL which leads to a lower number of circulating HDL particles. Furthermore, the HDL particles in NIDDM are smaller owing to a high hepatic lipase activity-another feature of NIDDM. Hepatic lipase has a great avidity for triglyceride-rich HDL and hydrolyses the triglyceride in the HDL core, which leads to a smaller HDL particle size. Small dense HDL and LDL particles are components of the 
dyslipidaemia of NIDDM [15]. The lowered plasma HDL-c and elevated plasma triglyceride levels are features of NIDDM dyslipidaemia (TG/HDL-c ratio above 3) which is a reliable predictor of insulin resistance. Insulin resistance a syndrome that favours atherosclerosis and thus, CAD (figure 1). Relying on LDL-c or total cholesterol alone can be misleading. It is also proved that people with obesity, metabolic syndrome or diabetic lipid disorders often have raised triglycerides, low HDL-c and normal or closed to normal LDL-c. Free radicals controls oxygen transport, require for cytochrome P-450 activity, prostaglandin cascade, phagocytosis, blood pressure regulation and detoxication processes. Under certain normal conditions oxygen may accept only one electron (usually in the electron transport chain accepts four electron and get converted to water) and this results in the formation of oxygen derived free radical superoxide $\left(\mathrm{O}_{2}{ }^{-}\right)$which may initiate the chain reaction of free radical formation. Malanodialdehyde is stable products of lipid peroxidation. Malanodialdehyde levels are indicative of lipid peroxidation which is a oxidative degeneration of polyunsaturated fatty-acids. Malanodialdehyde is regarded as a marker of inflammation induced by free radical injury on membrane lipids. Diabetes contributes to atherosclerosis through various mechanisms such as accelerated formation of reactive oxygen species due to decreased in activity superoxide dismutase, glutathione peroxidase [16]. Some studies have shown that diabetes mellitus may result from oxidative injury to the islets due to free radical production catalyzed by decompartmentalized transition metals such as iron and copper [17]. Elevated levels of free radicals in NIDDM oxidize the lipoproteins. Oxidized lipoproteins particularly oxidized LDL is more atherogenic. Several studies have suggested that the LDL may oxidized in arterial wall and thus initiate atherosclerosis. In addition to this, insulin at physiological level has antiatherogenic effects in vasculature [18]. Many reports show that in NIDDM activity of insulin to induce vasodilation is low due to insulin resistance [19].

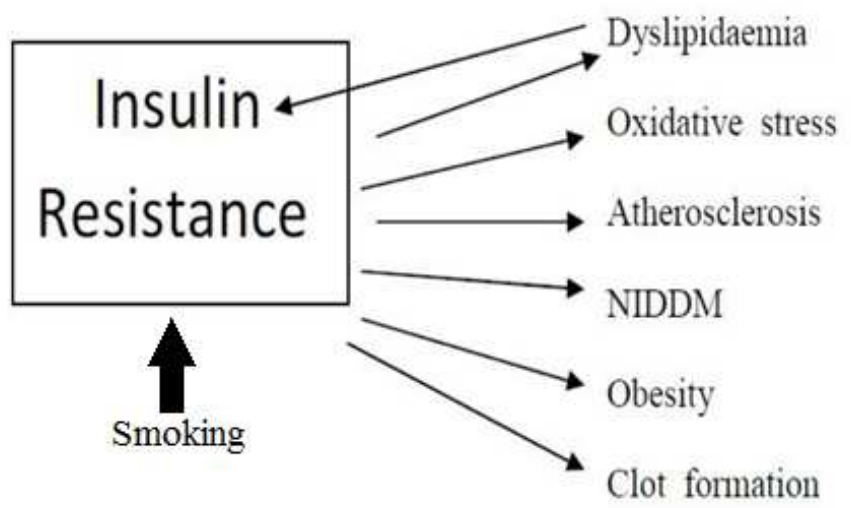

Figure-1: Biochemical and Pathological Consequences of Insulin Resistance

Smoking is other risk factors for CHD. In addition to inflammation, potential mechanisms by which smoking increases the risk of cardiovascular diseases include systemic haemostatic and coagulatory disturbances, lipid abnormalities, increase in oxidative stress, and vascular endothelial dysfunction [20]. Cigarette smoking leads to increase in the concentration of serum total cholesterol, LDL-c, triglycerides, VLDL-c, and fall in the levels of anti- atherogenic HDL-c [21]. It is presumed that nicotine stimulates sympathetic adrenal system leading to increased secretion of catecholamine resulting in increased lipolysis and increased concentration of plasma free fatty acids, which further results in increased synthesis of hepatic triglycerides, along with VLDL-c in the blood stream [22]. High levels of LDL-c, VLDL-c, and triglycerides are strongly associated with the development of coronary artery disease, while a low level of HDL-c remains a significant independent predictor of coronary artery disease [23]. Hypertension is one of the major risk factors for CHD. Hypertension is an independent risk factor for atherosclerosis. It is well known that hypertension is associated with abnormal changes in lipid profile (dyslipidemia) which is a cause for atherosclerosis [24]. Cigarette smoking NIDDM and hypertension individually potentiates lipid abnormalities but the combined effect has to be studied. Tobacco smoke contains large numbers of free radicals that are capable of initiating or promoting oxidative injury. Cigarette smokers have higher lipid peroxidation products in their blood when compared to nonsmokers and smoking increases the concentration of serum MDA levels [21]. It is suggested that oxidative injury may play a major role in mediating the health risks associated with cigarette smoking. Oxidation of LDL might be an important 
mechanism whereby cigarette smoking can accelerate atherogenesis. LDL from smokers was more susceptible to peroxidative modification when compared to that from non-smokers [21]. There is evidence to suggest that oxidized LDL may contribute to the pathogenesis of atherosclerosis [25]. NIDDM cigarette smoking and hypertension are proven independent risk factors for atherosclerosis; all these are associated with abnormalities in lipid profile and also show high values of serum MDA indicating increased rate of lipid peroxidation which suggests involvement of high rate of oxidative stress. We can assume that there is a cumulative effect of NIDDM, cigarette smoking and hypertension on these cardiovascular risk factors. From this study, it is well established that in NIDDM, cigarette smoking and hypertension there is dyslipidaemia and increased oxidative stress. The constellation of these altered lipoproteins along with lipid peroxides suggests that the patients with two or more risk factors are at a high risk for the development of coronary heart disease. There is a cumulative effect of NIDDM ,cigarette smoking and hypertension on lipid profile and lipid peroxidation, indicated by increased levels of serum MDA and dyslipidaemia indicated by higher values of total cholesterol, triglycerides, LDL-c, VLDL-c, and lower levels of cardio protective HDl-c. This observation suggests that there is a more risk of progression of atherosclerosis in individuals who have two or more risk factors i.e. NIDDM, smoking and hypertension. Further large population studies are required to substantiate our findings. In our study group triglycerides, VLDL-c, LDL-c and MDA positively related i.e significantly elevated as compared to healthy subjects. Rashida Meharan et. al and M. Deepa et. al reported that the diabetic dyslipidaemia and elevated free radicals (MDA) have role in advancement of atherosclerosis [26,27]. And Jeeyar, Hemalatha et.al reported the effect of smoking and hypertension on serum lipid profile and oxidative stress [28]. Observations of this present study are also correlated with these studies.

\section{Conclusion}

It can be concluded that patients of NIDDM with hypertension and smokers have higher total serum cholesterol, serum triglycerides, serum lipid peroxides (MDA), LDL- c and VLDL-c with lower HDL- c. These can be corrected, treated by dietary modification supplementation of vitamins, omega-3 fatty acids and life style modification before the development of end organ damage. For realization of exact role of lipid profile and oxidative stress further higher level studies should be done.

\section{Funding: Nil, Conflict of interest: None Permission of IRB: Yes}

\section{References}

1. Ross R. The pathogenesis of atherosclerosis--an update. N Engl J Med. 1986 Feb 20;314(8):488500.

2. Dewan BD, Malhotra KC, Gupta SP. Epidemiological study of coronary heart disease in rural community in Haryana. Indian Heart J. 1974 Apr;26(2):68-78.

3. Enas EA, Garg A and Davidson MA. Coronary artery disease and it's risk factors in $1^{\text {st }}$ generation immigrant Asian Indian to the United State of America. Indian Heart Journal 1996,48 (4)343-53.

4. Castelli WP and Andreson KA .Population at risk prevalence high cholesterol level in hypertensive patients Framingham study.American Journal of Medicine 1986, 80(2A) 23-28.

5. Bhoraskar AS and Raheja DS. Diabetes and cardiovascular disease Do Asian Indians have a high ethenic susceptibility. Journal of the Association of Physicians of India 1997,34 72-8.

6. Meigs JB, Singer DE and Sullivan LM. Metabolic control \&Prevalent cardiovascular disease in non- insulin dependent diabetes mellitus (NIDDM); The NIDDM patient outcomes research group. American Journal of Medicine 1997,102(1) $38-47$.

7. McLaughlin T, Abbasi F, Cheal K, Chu J, Lamendola C, Reaven G. Use of metabolic markers to identify overweight individuals who are insulin resistant. Ann Intern Med. 2003 Nov 18;139(10):802-9.

8. Kannel WB. Update on the role of cigarette smoking in coronary artery disease. Am Heart J. 1981 Mar;101(3):319-28.

9. Venkatesan A, Hemalatha A, Bobby Z, Selvaraj $N$, Sathiyapriya V. Effect of smoking on lipid profile and lipid peroxidation innormal subjects. Indian J Physiol Pharmacol 2006; 50(3): 273-278. 
10. Dincer Y, Akcay T, Aldemir Z and Likoova H . Effect of oxidative stress on Glutathione pathway in red blood cells from patients with insulindependent diabetes mellitus. Metabolism 2002, 51(10) 1360-1362.

11. Maritim AC, Sanders RA, Watkins JB 3rd. Diabetes, oxidative stress, and antioxidants: a review. J Biochem Mol Toxicol. 2003;17(1):24-38.

12. The American Diabetes association.Diagnosis and classification of diabetes mellitus.Diabetes Care. 2004;27 (supp 1) S5-S10.

13. Nandiger MA and Chandrakala MV . Estimation of serum total lipid peroxide (MDA). Malanodoaldehyde levels in different organs of rats, subjected to alcohol toxicity. Indian Journal of Clinical Biochemistry. 19861133.

14. Taskinen MR. Taskinen MR. Diabetes Metab Rev. 1987 Apr;3(2):551-70.

15. Taskinen MR, Lahdenperä S, Syvänne M. New insights into lipid metabolism in non-insulindependent diabetes mellitus. Ann Med. 1996 Aug;28(4):335-40.

16. Giugliano D, Ceriello A and Paolisso G . Diabetes mellitus, hypertension and cardiovascular disease and oxidative stress. Metabolism,1995, 44(3) 363-368.

17. Mukaopadhya .Free radicals and diabetes, Role in aetiology and pathogenesis Journal of Diabetic Association of India.1994, 34 5-7.

18. Feener EP, King GL. Vascular dysfunction in diabetes mellitus. Lancet. 1997 Jul;350 Suppl 1:SI9-13.

19. Velloso LA, Folli F, Sun XJ and White MF. Cross talk between the Insulin and angiotensin signaling systems. Proceedings of the National Academy of Sciences U.S.A,1996. 93,(22), 124905.
20. Yanbaeva DG, Dentener MA, Creutzberg EC, Wesseling G,Wouters EFM. Systemic effects of smoking. Chest 2007; 131(5),1557-1566.

21. Pasupathi P, Rao YY, Farook J, Saravanan G, BakthavathsalamG. Effect of smoking on lipids and oxidative stress biomarkers in patients with acute myocardial infarction. Res J Med Med Sci,2009,4(2)151-159.

22. Benowitz NL. Drug therapy. Pharmacologic aspects of cigarette smoking and nicotine addiction. N Engl J Med. 1988 Nov 17;319(20):1318-30.

23. Waqar A. Effect of tobacco smoking on the lipid profile of teenagemale population in Lahore City. Int J Med Med Sci 2010; 2(6):172-177.

24. Halperin RO, Sesso HD, Ma J, Buring JE, Stampfer MJ, Gaziano JM. Dyslipidemia and the risk of incident hypertension in men. Hypertension. 2006 Jan;47(1):45-50. Epub 2005 Dec 12.

25. Barbosa KB, Volp ACP, Hermsdorff HHM, Navarro-Blasco I, Zulet MA, Martínez JA, et al. Relationship of oxidized low density lipoprotein with lipid profile and oxidative stress markers in healthy young adults: a translational study. Lipids Health Dis.2011; Apr.19,10: 61.

26. Rashida Meharan, M. Mohsin, Zahida Nasreen, M. Siraj, and M. Ishaq. Significantly increased levels of serum malanodi aldehyde in type 2 diabetics with myocardial infraction. Int. J. Diabetes Dev. Ctries. 2010 Jan-Mar; 30(1):49-51.

27. Mathiyalagan Deepa, Palanisamy Pasupathi , K.B. Vidhya Sankar, P. Rani and S.P. Satish Kumar. Free radicals andantioxidant status in acute myocardial infarction patients with and without diabetes mellitus. Bangladesh Med Res Counc Bull 2009; 35(3), 95-100

28. Jeeyar, Hemalatha Wilma Delphine Silvia C.R.. Evaluation of effect of smoking and hypertension on serum lipid profile and oxidative stress. Asian Pacfic J. of Tropical disease. 2011,289-291.

\section{How to cite this article?}

Suresh S. Ugle, Mahendra D. Bikkad. Role of lipids and free radicals in diabetic with hypertension and smokers for causation of coronary heart disease. Int J Med Res Rev 2017;5(06):620-625. doi:10.17511/ijmrr. 2017.i06.12. 